\title{
A Call to Action: Intensify the Investigation of Relatively Rare Tumors of the Gastrointestinal Tract
}

\author{
Lei Shen Jing-Yuan Fang \\ Division of Gastroenterology and Hepatology, Renji Hospital, Shanghai Jiao Tong University \\ School of Medicine, Shanghai Institute of Digestive Disease, Shanghai, China
}

Recently, certain types of gastrointestinal tumors that were rarely diagnosed previously appear to be increasing in frequency. Gastrointestinal stromal tumors (GISTs) were rare in the twentieth century, and the term was used in confusion with leiomyomas and leiomyosarcomas. At the end of the last century, Hirota and others defined the histologic and molecular pathogenesis of GISTs. These tumors bear activated c-KIT gain-of-function mutations for the enzyme c-KIT tyrosine kinase, which may play a central role in their pathogenesis. The KIT receptor can be detected by immunohistochemical staining for CD117, which is the epitope on its extracellular domain [1].

As $70 \%$ of patients with GISTs are asymptomatic, the tumors are often accidentally detected during endoscopic examination or surgical operations. In practice, $90 \%$ of GIST cases show CD117 positivity. Other GISTs express PDGFR or show different gene mutations. Thus, the etiology and pathogenesis of GISTs involve certain complex molecular changes.

According to the data from American Surveillance, Epidemiology, and End Results (SEER), 4,411 GIST cases were diagnosed in the 1990-2009 period, in locations including the esophagus $(29 / 4,411)$, stomach $(2,858 / 4,411 ; 65 \%)$, small intestine $(1,463 / 4,411 ; 33 \%)$, colon $(126 / 4,411)$ and rectum $(135 / 4,411)$ [2]. In clinical practice, endoscopic ultrasonography (EUS) can frequently detect submucosal tumors of the esophagus, but the majority of such tumors are CD117-negative and are true leiomyomas, not GISTs. The colon and rectum are uncommon sites for GIST occurrence, and tumors occurring here may be detected during endoscopic examination.

The annual age-adjusted incidence of GISTs (calculated from 6,142 CD117-positive cases occurring within the $2001-2011$ period) was $0.55 / 100,000$ in 2001 and rose to $0.78 / 100,000$ in 2011. The GIST incidence increased with age, peaking within the 70-79 years group $(3.06 / 100,000)$. The most common age range at the time of diagnosis was 50-60 years. The study examined the 5-year survival and showed GIST-specific survival rates of 65 and $79 \%$ 
[3]. A study by Chiang et al. [4] from Taiwan showed that the incidence of GISTs in the 20052008 period was $1.97 / 100,000$, the median survival being 8.4 years.

Computed tomography scans are effective at imaging GIST lesions. EUS is useful for the detection of submucosal masses, and GISTs can be detected via EUS as a hypoechoic mass arising from the second or fourth echo layer. Putative EUS criteria to differentiate benign from malignant GISTs include a tumor size $>4 \mathrm{~cm}$, an irregular extraluminal margin and a cystic space $>4 \mathrm{~mm} .{ }^{18} \mathrm{~F}$-FDG-PET can detect lesions that are around $1 \mathrm{~cm}$ in size, because neither the normal bowel or omentum will take up the ${ }^{18}$ F-FDG tracer.

The treatment of GISTs can involve several approaches. Tumors with a diameter $<1.0 \mathrm{~cm}$ are usually observed periodically. Larger GISTs can be removed using endoscopy or surgery. Advanced GISTs with metastases that cannot be removed can be targeted using tyrosine kinase inhibitors (imatinib mesylate, $400 \mathrm{mg} /$ day). However, the optimal dose and duration of imatinib treatment has not been determined, and imatinib-resistant disease may emerge after 1-2 years of a durable response due to clonal evolution of the GIST. It is now clear that administration of imatinib in post-resection settings has the potential to delay GIST recurrence. The functional imaging of GISTs with ${ }^{18} \mathrm{~F}$-FDG-PET can provide useful information in the management of GIST patients.

The entity of GIST has been established only for the past 20 years, and now it is the most common mesenchymal tumor of the gastrointestinal tract [5]. Personalizing the treatment of GISTs in order to reach better follow-ups and outcome results is an attractive strategy.

Neuroendocrine tumors (NETs) of the gastrointestinal tract are neoplasms of enterochromaffin/neuroendocrine cell origin that display neuroendocrine capacity. NETs can include gastrointestinal NETs and pancreatic NETs. Gastrointestinal NETs have been termed 'carcinoid tumors' for over a century. In comparison with colorectal cancer, relatively little is known about the molecular events underlying the evolution of the small intestine tumors $[6,7]$.

Modlin et al. [8] carried out a five-decade analysis of carcinoid tumors in the United States and reported that the estimated incidence varied from 2.5 to 5 cases per 100,000 individuals. A European investigation that included both surgical and autopsy specimens reported an overall incidence of 8.4 cases per 100,000 individuals [8]. However, incidence estimations are limited by the clinically silent nature of many NETs, which remain undetected until autopsy. $67 \%$ of NETs occur in the gastrointestinal tract, while $25 \%$ occur in the bronchopulmonary system.

Moreover, the available data indicate that NETs are undergoing a location change within the gastrointestinal tract, with this shift in the anatomic location occurring over the last halfcentury. Data from 1950 to 1971 identified the appendix as the most common site of gastrointestinal NETs, followed by the rectum and ileum. A recent evaluation of NETs between 1973 and 1999 found that the small intestine was the most frequent site $(41 \%)$ for gastrointestinal NETs, followed by the rectum, while only $4.8 \%$ of cases occurred in the appendix. Additionally, gastric NETs account for an increasing proportion of gastrointestinal NETs, increasing from 2.4 to $8.7 \%$. NETs are now the most common malignancy of the small intestine. The observed change in sites of occurrence may be due to the widespread use of endoscopy, computed tomography and magnetic resonance imaging as diagnostic instruments. Alternatively, some investigators have suggested that treatment of acid-related diseases using proton pump inhibiters may stimulate the proliferation of enterochromaffin/neuroendocrine cells and promote the formation of NETs. However, no conclusion can be reached at this point [9].

The median age at diagnosis of gastrointestinal NETs is 60 years. $90 \%$ of small intestine carcinoid tumors are found in the distal ileum. Despite their indolent course, NETs are malignant, and approximately one-third of patients will have more than one tumor. At the time of NET diagnosis, $12.9 \%$ of patients have metastasis [10]. NET cells secrete more than 
40 substances, including 5-hydroxytryptamine, gastrin, histamine, substance $\mathrm{P}$, chromogranin A and prostaglandins. Although many kinds of biomarkers have been tested, biomarkers with satisfactory sensitivity and specificity are lacking for early and accurate diagnosis of gastrointestinal NETs.

The most common clinical symptom is a history of prolonged nonspecific abdominal pain; other common symptoms include occult or frank bleeding, intussusceptions and flushing diarrhea. Duodenal carcinoid tumors as small as $2-5 \mathrm{~mm}$ can be detected by EUS, and determination of invasion with EUS can be useful for staging.

Resection is the only curative therapy for small intestinal NETs; pharmacological interventions involving the use of somatostatin, octreotide and interferons have not shown consistent effects and require further study.

Since the incidences of GISTs and NETs of the gastrointestinal tract is increasing, with a low 5-year survival rate and a lack of effective diagnostic tools and treatments, further investigation and development of strategies is critically needed. From this standpoint, pathoanatomy, which has greatly contributed to medical progress in the last century, may be useful in managing difficult and resistant cases of GISTs and NETs, as it may allow a deeper understanding of pathogenesis and result in an improvement in outcomes.

\section{References}

1 Rammohan A, Sathyanesan J, Rajendran K, Pitchaimuthu A, Perumal SK, Srinivasan U, Ramasamy R, Palaniappan R, Govindan M: A gist of gastrointestinal stromal tumors: a review. World J Gastrointest Oncol 2013;5:102112.

-2 Kukar M, Kapil A, Papenfuss W, Groman A, Grobmyer SR, Hochwald SN: Gastrointestinal stromal tumors (GISTs) at uncommon locations: a large population based analysis. J Surg Oncol 2015;111:696-701.

-3 Ma GL, Murphy JD, Martinez ME, Sicklick JK: Epidemiology of gastrointestinal stromal tumors in the era of histology codes: results of a population-based study. Cancer Epidemiol Biomarkers Prev 2015;24:298-302.

$>4$ Chiang NJ, Chen LT, Tsai CR, Chang JS: The epidemiology of gastrointestinal stromal tumors in Taiwan, 19982008: a nation-wide cancer registry-based study. BMC Cancer 2014;14:102.

5 Kramer K, Wolf S, Mayer B, Schmidt SA, Agaimy A, Henne-Bruns D, Knippschild U, Schwab M, Schmieder M: Frequency, spectrum and prognostic impact of additional malignancies in patients with gastrointestinal stromal tumors. Neoplasia 2015;17:134-140.

-6 Salyers WJ, Vega KJ, Munoz JC, Trotman BW, Tanev SS: Neuroendocrine tumors of the gastrointestinal tract: case reports and literature review. World J Gastrointest Oncol 2014;6:301-310.

7 Chen YX, Fang JY: Current perspective on the pathogenesis of small intestinal neuroendocrine tumors: progress in biomarkers and molecular events. Gastrointest Tumors 2014;1:2-8.

8 Modlin IM, Lye KD, Kidd M: A 5-decade analysis of 13,715 carcinoid tumors. Cancer 2003;97:934-959.

-9 Modlin IM, Lye KD, Kidd M: A 50-year analysis of 562 gastric carcinoids: small tumor or large problem? Am J Gastroenterol 2004;99:23-32.

10 Bresalier RS, Blechacz B: Tumors of the small intestine; in Feldman M, Friedman LS, Brandt LJ (eds): Sleisenger and Fordtran's Gastrointestinal and Liver Disease, ed 10. Philadelphia, Elsevier Saunders, 2010, pp 21962212. 